\title{
Non-Fermenting Gram Negative Bacteria as Uropathogens in Causing Urinary Tract Infection and its Antimicrobial Susceptibility Pattern at A Tertiary Care Centre of South India
}

\author{
Anupam Berwal ${ }^{1}(\mathbb{D})$ K.L. Shobha ${ }^{1 *}$ (D) Rohit Gupta $^{1}$, Kriti Gupta², \\ Asem Ali Ashraf ${ }^{1}$, Abishek Kumar B ${ }^{1}$, Raghav Chawla ${ }^{1}$, Prerna Aggarwal ${ }^{3}$, \\ Bhawna Sharma ${ }^{3}$ and Sonali ${ }^{4}$ \\ ${ }^{1}$ Department of Microbiology, Kasturba Medical College, Manipal, Manipal Academy Of Higher Education, \\ Manipal, Karnataka, India. \\ ${ }^{2}$ Dr. Niraj Kumar Gupta MicroPath Lab, Bijnor (U.P.) India. \\ ${ }^{3}$ Department of Microbiology, Kalpana Chawla Govt. Medical College, Karnal, India. \\ ${ }^{4}$ Department of Pathology, Kalpana Chawla Govt. Medical College, Karnal, India.
}

\begin{abstract}
Non fermenting gram-negative bacilli (NFGNB) are recently striving as uropathogens. The present study was conducted to isolate the common species of bacteria in NFGNB causing urinary tract infection (UTI) and its correlation with comorbid conditions and to study the antibacterial susceptibility pattern. This retrospective study was done at the diagnostic Microbiology laboratory of a tertiary care hospital. Urine samples were collected for the period of six months. These samples were plated on blood agar and MacConkey agar and incubated at $37^{\circ} \mathrm{C}$ for $18-24 \mathrm{hr}$ under aerobic conditions. Identification of NFGNB was done by Gram staining and MALDI-TOF (Matrix- Assisted Laser Desorption/ lonizationTime of Flight, Biomerieux- Diagnostics). Antibiotic sensitivity testing was done by Vitek ${ }^{\circledR} 2$ system (Biomerieux- Diagnostics) using N 281 card. Data was analyzed using SPSS IBM version 16. Out of the total 16,413 non repetitive urine samples that were received in the laboratory, 318 had significant bacteriuria. NFGNB were identified in 108 (33.9\%) of all the urine samples with significant bacteriuria. Prevalence of non-fermenters in our study was $0.6 \%$. NFGNB were more frequently isolated in the females and also in the age group of more than 50 years. Eighty five $\mathbf{( 7 8 . 7 0 \% )}$ had comorbid conditions. $P$. aeruginosa and $A$. baumannii were the most common organism isolated among NFGNB. Pseudomonas aeruginosa isolates showed high susceptibility to imipenem $(80.2 \%)$ and amikacin $(66.6 \%)$. NFGNB although seen frequently in females and in age group of $\mathbf{5 0}$ years and above, clinical correlation with comorbid condition is essential to label it as uropathogens. Amikacin or imipenem may be the empirical drug of choice.
\end{abstract}

Keywords: NFGNB, comorbid condition, Antibiotic susceptibility, Bacteriuria, Urinary tract infection

*Correspondence: shobha.kl@manipal.edu; 9945271648

(Received: March 10, 2020; accepted: August 07, 2020)

Citation: Berwal A, Shobha KL, Gupta R et al. Non-Fermenting Gram Negative Bacteria as Uropathogens in Causing Urinary Tract Infection and its Antimicrobial Susceptibility Pattern at A Tertiary Care Centre of South India. J Pure Appl Microbiol. 2020;14(3):2033-2038. doi: 10.22207/JPAM.14.3.43

(C) The Author(s) 2020. Open Access. This article is distributed under the terms of the Creative Commons Attribution 4.0 International License which permits unrestricted use, sharing, distribution, and reproduction in any medium, provided you give appropriate credit to the original author(s) and the source, provide a link to the Creative Commons license, and indicate if changes were made. 


\section{INTRODUCTION}

Non fermenting gram-negative bacilli (NFGNB) are gram negative bacilli that can survive and grow in oxygenated environment and are incapable of fermenting glucose \& utilizing it as a sole source of energy ${ }^{1}$. Hospital surroundings and intestines of human beings provide a niche for them to survive as saprophytes ${ }^{2,3}$.

They may pose a serious problem by causing infections associated with indwelling medical devices like urinary catheter or ventilator. Around $12-16 \%$ of all bacterial species isolated from the laboratory are identified as nonfermenting gram negative bacilli ${ }^{4}$. High degree of resistance to various biocides are present in these isolates. Widespread patient-to-patient transmission occurs either via contaminated surfaces or through the hands of the health care providers especially in immunocompromised patients ${ }^{2,5}$. NFGNB include numerous organisms like Pseudomonas spp, Acinetobacter spp, Alkaligenes spp, Stenotrophomonas maltophilia, Burkholderia cepacia complex (BCC). Presently, $P$. aeruginosa and $A$. baumannii are the most frequently isolated non-fermenters ${ }^{6}$.

Factors that pose a risk of NFGNB infection include immunosuppression (cancer patients, organ transplant patients and AIDS), neutropenic patients, diabetes, trauma, foreign body like indwelling catheters, ventilators and implants, overuse and inappropriate consumption of broad-spectrum antibiotics, intravenous saline infusion ${ }^{7}$ and Foley's catheter.

Multidrug resistance among NFGNB is very common and is constantly rising. Carbapenem resistance among Pseudomonas and Acinetobacter leaves very less treatment options ${ }^{8}$. Carbapenemase activity in $A$. baumannii is mainly due to carbapenem-hydrolyzing class $D$ b-lactamases (CHDLs) and in case of $P$. aeruginosa the predominant mechanism of resistance to carbapenem is loss of OprD2 which is a substratespecific protein molecule that facilitates the diffusion of carbapenems into the cell ${ }^{9}$. Recently few studies conducted in India have identified the antimicrobial sensitivity pattern for these organisms $\mathrm{s}^{3,10,11}$.

Other organisms usually were ignored and were conveniently presumed to be contaminants because identification of species of these organisms is time-taking and hence very difficult in a busy routine diagnostic centre ${ }^{12}$. But introduction of the automated identification systems like Vitek 2 Compact and MALDI-TOF has made this task possible.

NFGNB have now been given a status of more than just a contaminant because of their increased isolation from urine samples with significant bacteriuria indicating their pathogenic role in causing UTI. Clinical correlation is required in order to label them either as colonizer or as pathogen ${ }^{13-15}$.

Rationale of the Study: To isolate various species of NFGNB causing UTI, its associated comorbid conditions and their antimicrobial susceptibility pattern in the recent years.

\section{MATERIALS AND METHODS}

Urine specimens collected from patients attending tertiary care hospital for routine culture and susceptibility test over a study period of 6 months were included in the present study. The urine samples included midstream urine samples, urine from Foley's catheter or from DJ stenting. These specimens were processed by culturing on $5 \%$ sheep blood agar and MacConkey agar. Those midstream urine samples yielding a monomicrobial culture of $\geq 10^{5} \mathrm{CFU} / \mathrm{ml}$ were noted as cases having significant bacteriuria according to Kass concept, while any number of colonies in samples collected from Foley's catheter or DJ stenting were considered significant. Identification was done by Gram staining and MALDI-TOF. Antibiotic susceptibility testing was done by Vitek ${ }^{\circledR} 2$ system using N 281 card. The antibiotics used for sensitivity testing were piperacillin-tazobactam, ceftazidime, cefepime, cefoperazone-sulbactam, imipenem, meropenem, aztreonam, amikacin, gentamycin, ciprofloxacin, trimethoprim-sulfamethoxazole, tigecycline and colistin.

Details of the patients such as demography, signs and symptoms, radiological impression, ICU/ward admission, hospitalisation and prognostic outcomes were retrospectively collected from the medical record department. The case sheet of individual patients were analysed for relevant laboratory and clinical criteria in order to access the clinical significance of the isolates. The criteria in the laboratory for the diagnosis of 
UTI caused by NFGNB included the presence of pus cells along with gram-negative bacilli in the stained smear from the sample, monomicrobial infection, isolation of the same organism from a repeat sample, raised total leucocyte count, and significant radiological findings (in patients with complicated UTI)5. The clinical criteria included burning micturition, lower abdominal pain, pyuria, ureteric stone and urinary retention.

\section{Statistical analysis}

Statistical analysis was done using software SPSS version 16 .

\section{RESULTS}

Among the total number of 16,413 urine samples that were received in the laboratory, 318 had significant bacteriuria. NFGNB were isolated in 108 (33.9\%) the urine samples with significant bacteriuria. Prevalence of non-fermenters out of the total urine samples collected was $0.6 \%$.

It was found that all the samples that grew NFGNB were from the patients who were admitted in the hospital. Maximum number of isolates were found from patients within the age range of 51-60 Years (Table 3). Ratio of males, $44(40.47 \%$,) to females $64(59.25 \%)$ from which NFGNB were isolated was 1:1.55 (Table 2). Seven (6.5\%) out of 108 samples, were from pregnant women. Previous hospitalization was from 43 cases (39.8\%). Nine samples (8.3\%) were from the patients admitted in ICU.

Among the NFGNB cases, samples collected from the mid-stream urine were 75(69.4\%), indwelling catheter (Foley's catheter) were $19(17.6 \%)$ and samples from DJ stent were 14 (12.9\%). Pyuria was seen in $63(58.3 \%)$ patients and recurrent UTI was a complaint in $38(35.1 \%)$ cases. Among the comorbid conditions, patients with diabetes were $27(25 \%)$, hypertension were 35 (32.4\%), chronic kidney disease were 12(11.1\%), and immunosuppression were $11(10.2 \%)$. Total number of patients with comorbid conditions were 85/108 (78.70\%) Out of 108 patients in which NFGNB were isolated, three patients did not survive. Rest all were successfully treated with antibiotics.

Pseudomonas aeruginosa was the most predominant isolate growing in 77 (71.29\%) urine samples followed by Acinetobacter spp $22(20.37 \%)$.

\section{DISCUSSION}

Out of 16,413 urine samples that were received in the laboratory, 318 had significant bacteriuria. NFGNB were isolated in 108 (33.9\%) of the 318 urine samples with significant growth.

Table 1. Antibiotic susceptibility pattern of NFGNB isolated in the study

\begin{tabular}{lccc}
\hline Antibiotics & $\begin{array}{c}\text { Pseudomonas spp } \\
(\mathrm{n}=81) \text { Sensitivity \% }\end{array}$ & $\begin{array}{c}\text { Acinetobacter spp } \\
(\mathrm{n}=22)\end{array}$ & $\begin{array}{c}\text { Sensitivity \% } \\
(\mathrm{n}=5) \text { Sensitivity \% }\end{array}$ \\
\hline Amikacin & 66.67 & 63.64 & - \\
Ceftazidime & 64.20 & 0.09 & - \\
Ciprofloxacin & 51.90 & 31.82 & - \\
Gentamicin & 58.02 & 27.27 & - \\
Aztreonam & 20.99 & 0 & - \\
Cefoperazone- & 62.96 & 63.64 & - \\
sulbactum & & & - \\
Cefepime & 72.84 & 63.64 & - \\
Imipenem & 80.25 & 77.27 & \\
Meropenem & 91.36 & 90.91 & 100 \\
Piperacillin- & 82.70 & 63.64 & \\
Tazobactam & 88.89 & 95.45 & \\
Tigecycline & 100 & 100 & \\
Colistin & - & - & \\
Trimethoprim- & & & \\
sulfamethoxazole & & & \\
\hline
\end{tabular}

Pseudomonas spp included Pseudomonas aeruginosa $(\mathrm{n}=77)$ and Pseudomonas putida $(\mathrm{n}=4)$.

Acinetobacter spp included Acinetobacter baumanii complex $(\mathrm{n}=21)$ and Acinetobacter junii $(\mathrm{n}=1)$. (-) indicates not applicable. 
Table 2. Gender distribution of patients in which NFGNB were isolated

\begin{tabular}{lccc}
\hline Gender & $\begin{array}{c}\text { Pseudomonas spp. } \\
(n=81)\end{array}$ & $\begin{array}{c}\text { Acinetobacter spp. } \\
(n=22)\end{array}$ & $\begin{array}{c}\text { Stenotrophomonas maltophilia } \\
(n=5)\end{array}$ \\
\hline Females & & & \\
$(64) 59.25 \%$ & 54 & 7 & 3 \\
Males (44) 40.74\% & 27 & 15 & 2 \\
\hline
\end{tabular}

Table 3. Demographic details of patients in which NFGNB were isolated

\begin{tabular}{lccc}
\hline $\begin{array}{l}\text { Age group } \\
\text { (in years) }\end{array}$ & $\begin{array}{c}\text { Pseudomonas } \\
\text { spp. }\end{array}$ & $\begin{array}{c}\text { Acinetobacter } \\
\text { spp. }\end{array}$ & $\begin{array}{c}\text { Stenotrophomonas } \\
\text { malophilia }\end{array}$ \\
\hline$>=10$ & 2 & 3 & 0 \\
$11-20$ & 4 & 2 & 0 \\
$21-30$ & 9 & 2 & 0 \\
$31-40$ & 6 & 1 & 0 \\
$41-50$ & 5 & 1 & 0 \\
$51-60$ & 15 & 6 & 1 \\
$61-70$ & 11 & 3 & 2 \\
$71-80$ & 14 & 4 & 2 \\
$81-90$ & 15 & 0 & 0 \\
Total no. & 81 & 22 & 05 \\
of isolates & & & \\
\hline
\end{tabular}

Out of the 108 patients, females were $59.25 \%$ and males $40.74 \%$. A similar study conducted by Rachana Solanki et al. ${ }^{16}$ in Kolar, Karnataka reported $1.6 \%$ NFGNB. In the previous study done by Shobha KL et al. ${ }^{17}$ at Manipal, out of 1271 midstream urine isolates with significant bacteriuria, NFGNB were found in 120 (9.44\%) cases and was higher in female patients. Higher isolation rate of non-fermenters in our study may be attributed to the fact that majority of the samples collected in our study were from patients who were admitted in the hospital either in ward or ICU due to some underlying causes and were on Foley's catheter or DJ stenting.

Among 108 patients from which NFGNB were isolated, $67.6 \%$ were above fifty years of age. This finding was in concordance to a study done in Telangana where $69.04 \%$ NFGNB were isolated in the age group of $>50$ years of age.

In the present study, $71.3 \%$ of the nonfermenters were identified as $P$. aeruginosa which was similar to the study done by Rachana Solanki et al. ${ }^{16}$ in Kolar, Karnataka where the same organism was isolated in $56.5 \%$ urine samples. A similar finding was found in a study conducted in Hungary where Pseudomonas spp. (outpatients:
78.7\%; inpatients:85.1\% ) and Acinetobacter spp. (outpatients: $19.6 \%$, inpatients: $10.9 \%$ ), were the most prevalent organisms isolated ${ }^{19}$.

NFGNB that were earlier considered to be contaminants have been implicated in causing many health care associated infection $\mathrm{s}^{20}$. $P$. aeruginosa and Acinetobacter species were most commonly associated with such health care associated infections ${ }^{20}$. Present study showed Pseudomonas species and Acinetobacter species accounted for $95.3 \%$ of the isolates. They comprised $87 \%$ of the total non-fermenter isolated in the study done in Kolar, Karnataka ${ }^{3}$.

Antimicrobial susceptibility pattern of one region differs from other; further it changes with time according to the adaptability of the infecting organism ${ }^{21}$. High grade of susceptibility to imipenem (80.2\%) and amikacin (66.6\%) was found in our Pseudomonas isolates which were similar to the study done in Kolar, Karnataka3 where susceptibility to imipenem was $94 \%$ and to amikacin was $69 \%$. In a study conducted at Bangalore, $60-70 \%$ isolates of $P$. aeruginosa showed resistance to amikacin, ceftazidime, and ciprofloxacin ${ }^{22}$. In a similar study done at Chandigarh $42 \%$ of $P$. aeruginosa isolates showed 
resistance to imipenem ${ }^{23}$. Most of the patients in our study were admitted in wards, while very few of them needed ICU care. Pseudomonas aeruginosa isolates were fairly sensitive to imipenem, amikacin, and cefoperazone. A. baumannii were sensitive to imipenem and piperacillin.

NFGNB though earlier regarded as contaminants have now being increasingly implicated in causing both hospital- and community-acquired infection. In our study, patients with comorbid conditions were 85/108 (78.70\%) who definitely needed treatment for urinary tract infection due to NFGNB infection, where NFGNB is uropathogen. Therefore clinical correlation is necessary to determine this pathogenic role of NFGNB before ignoring them as mere contaminant. Identification of these organisms as uropathogens with assessment of their antibiotic sensitivity is necessary for appropriate treatment of this infection .

\section{CONCLUSION}

Pseudomonas aeruginosa and Acinetobacter baumannii were the most frequent non-fermenters isolated in our study. Though NFGNB are important etiological agents causing urinary tract infection more frequently in females and in the age group of $>50$ years, the clinical correlation with comorbid conditions of the patients should be made to identify NFGNB as uropathogen. This would prevent the use of unnecessary antibiotics in patients having NFGNB as colonizer and emergence of drug-resistant strains. Amikacin or imipenem may be the empirical drug of choice in these cases.

\section{ACKNOWLEDGMENTS}

None.

\section{CONFLICT OF INTEREST}

The authors declare that there is no conflict of interest.

\section{AUTHORS' CONTRIBUTIONS}

SKL: Planned the study and helped in editing the manuscript. $A B$ and RG: Conducted the study and manuscript writing. AKB and RC: Helped in data entry. KG and AAA: Manuscript writing and ethical approval of study.

\section{FUNDING}

None.

\section{ETHICS STATEMENT}

Ethics approval was taken and ethics approval number is IEC: 349/2019.

\section{DATA AVAILABILITY}

All datasets generated of analyzed during this study are included in the manuscript.

\section{REFERENCES}

1. Makkar A, Panda PS, Khan ID, Banerjee P, Kaira SS, Rajmohan KS. Non- Fermenters as Mysterious Pathogens or Contaminants- Continuing Dilemma Saudi J Pathol Microbiol. 2018;3(9):303-309.

2. Steinberg JP, Rio DC. Gram negative and Gram variable bacilli. In: Mandell GL, Bennett JE, Dolin R, editors. Principles and Practice of Infectious diseases. $6^{\text {th }}$ ed. Philadelphia, USA: Elsevier Publication. 2005;2:27512768.

3. Malini A, Deepa EK, Gokul BN, Prasad SR. Nonfermenting gram-negative bacilli infections in a tertiary care hospital in kolar, karnataka. J Lab Physicians. 2009;1(2):62-66. doi: 10.4103/09742727.59701

4. Grewal US, Bakshi R, Walia G, Shah PR. Antibiotic susceptibility profiles of non-fermenting gramnegative Bacilli at a Tertiary Care Hospital in Patiala, India. Niger Postgrad Med J. 2017;24(2):121-125. doi: 10.4103/npmj.npmj_76_17

5. Quinn JP. Clinical problems posed by multiresistant nonfermenting gram-negative pathogens. Clin Infect Dis. 1998;1;27(Supplement_1):S117-24. doi: $10.1086 / 514912$

6. Chawla K, Vishwanath S, Munim FC. Nonfermenting Gram-negative Bacilli other than Pseudomonas aeruginosa and Acinetobacter Spp. Causing Respiratory Tract Infections in a Tertiary Care Center. J Glob Infect Dis. 2013;5(4):144-148. doi: 10.4103/0974$777 X .121996$

7. Hall GS. Non fermentative gram-negative bacilli and miscellaneous gram negative rods. In : Textbook of diagnostic Microbiology. Conneie R Mahan. George Manuselis Jr. eds. Philadelphia. 1995:513-538.

8. Krishnan S, Santharam P, Shanmugavadivoo N, Usha B. Prevalence of Non-Fermenting Gram Negative Bacilli and Their Antibiotic Sensitivity Pattern at a Tertiary Care Hospital in Tamilnadu. Int J Curr Microbiol App Sci. 2018;7(2):2751-2758. doi: 10.20546/ ijcmas.2018.702.335

9. Quinn JP, Studemeister AE, DiVincenzo CA, Lerner SA. Resistance to imipenem in Pseudomonas aeruginosa: Clinical experience and biochemical mechanisms. Rev Infect Dis. 1988;10(4):892-898. doi: 10.1093/ clinids/10.4.892

10. Gautam V, Ray P, Vandamme P, et al., Identification of lysine positive non-fermenting gram negative bacilli (Stenotrophomonas maltophilia and 
Burkholderiacepacia complex). Indian J Med Microbiol. 2009;27:128-133. doi: 10.4103/0255-0857.49425

11. Samanta P, Gautam V, Thapar R, Ray P. Emerging resistance of non-fermenting gram negative bacilli in a tertiary care centre. Indian J PatholMicrobiol. 2011;54(3):666-667. doi: 10.4103/0377-4929.85150

12. Stansfeld JM. Clinical observations relating to incidence and aetiology of urinary-tract infections in children. BMJ. 1966;1(5488):631. doi: 10.1136/bmj.1.5488.631

13. Gupta V, Yadav A, Joshi RM. Antibiotic resistance pattern in uropathogens. Indian J Med Microbiol. 2002;20(2):96.

14. Mammeri H, Bellais S, Nordmann P. Chromosomeencoded $\beta$-lactamases TUS- 1 and MUS- 1 from Myroides odoratus and Myroides odoratimimus (formerly Flavobacterium odoratum), new members of the lineage of molecular subclass B1 metalloenzymes. Antimicrob Agents Chemother. 2002;46(11):35613567. doi: 10.1128/AAC.46.11.3561-3567.2002

15. Duggan JM, Goldstein SJ, Chenoweth CE, Kauffman CA, Bradley SF. Achromobacter xylosoxidans bacteremia: report of four cases and review of the literature. Clin Infect Dis. 1996;23(3):569-576. doi: 10.1093/ clinids/23.3.569

16. Solanki R, Dawar R, Aggarwal DK, Rani H, Imdadi F, Jasusa S. Nonfermenting Gram-Negative Bacilli and Urinary Tract Infection-Sorting the Mystery of Infections Caused. J Med Microb Diagn. 2015;4(4):2161-0703. doi: 10.4172/2161-0703.1000210

17. Shobha KL, Rao GG, Kukkamalla AM. Prevalence Of Non-fermenters In Urinary Tract Infections In A Tertiary
Care Hospital. www.webmedcentral.com/wmcpdf/ Article_WMC001464.pdf.

18. Yeruva $S$, Kabra V. Identification and Antimicrobial Susceptibility testing of Non Fermenting Gram Negative Bacteria by Vitek 2 in a Teaching Hospital in Mahbubnagar. Int J Curr Microbiol App Sci. 2018;7(10):234-240. doi: 10.20546/ijcmas.2018.710.024

19. Gajdacs M, Burian K, Terhes G. Resistance levels and epidemiology of non-fermenting gram-negative bacteria in urinary tract infections of inpatients and outpatients (RENFUTI): a 10-year epidemiological snapshot. Antibiotics. 2019;8(3):143. doi: 10.3390/ antibiotics 8030143

20. Troillet N, Samore MH, Carmeli Y. Imipenem-Resistant Pseudomonas aeruginosa: Risk factors and Antibiotic susceptibility patterns. Clin Infect Dis. 1997;25:10941098.doi: 10.1086/516092

21. Prashanth $\mathrm{K}$, Badrinath $\mathrm{S}$. In vitro susceptibility pattern of Acinetobacter species to commonly used cephalosporins, quinolones, and aminoglycosides. Indian J Med Microbiol. 2004;22(2):97.

22. Kumari HV, Nagarathna S, Chandramuki A. Antimicrobial resistance pattern among aerobic gram-negative bacilli of lower respiratory tract specimens of intensive care unit patients in a neurocentre. Indian Journal of Chest Diseases and Allied Sciences. 2007 Jan 30;49(1):19.

23. Taneja N, Maharwal S, Sharma M. Imipenem resistance in nonfermenters causing nosocomial urinary tract infections. Indian J Med Sci. 2003;57:294-299. 